\title{
An Exploratory Study of Touch Zones in College Students on Two Campuses
}

\author{
Mark Tomita \\ California State University, Chico
}

\begin{abstract}
The purpose of this exploratory study was to characterize college student beliefs about where it is acceptable to touch and be touched by other students in casual social interactions. Undergraduate students at a residential university $(\mathrm{N}=242)$ and at a local community college $(\mathrm{N}=200)$ completed the Touch Survey. The survey measures beliefs about touching in social interactions. Hierarchical cluster analyses were used to form touch zones (Public, Discretionary, and Private) by gender and direction of touch. The results of the study showed distinct same- and opposite-gender touch zone patterns, and there were touch zone differences between the two campuses. There were reciprocal touch zones for residential university male/male public touch zones and female/female private touch zones. There were no reciprocal touch zones for the community college sample. Implications for college health educators are discussed.
\end{abstract}

(c) 2008 Californian Journal of Health Promotion. All rights reserved.

Keywords: college students, touch, sexual harassment, social interaction

\section{Introduction}

Touch, or tactile communication, is a healthy and essential part of interpersonal communication where cultural beliefs and attitudes influence these social health behaviors (Dibiase \& Gunnoe, 2004; Floyd, 2000; Remland, Jones, Brinkman, 1995). Some touches, however, may be interpreted as sexually harassing. Many students come to college already having had negative experiences with sexual harassment. In a national study of high school students, for example, as many as $83 \%$ of girls and $79 \%$ of boys reported having ever experienced sexual harassment [American Association of University Women Educational Foundation (AAUW), 2001]. Of these students, almost a fourth defined sexual harassment as unwanted touch. According to a recent national study (Hill and Silva, 2006), a third of college freshman have been sexually harassed. Eighty nine percent of students say sexual harassment occurs on their campus, and $83 \%$ of students say they would be very or somewhat upset about someone touching (e.g., touch, pinch, or grab) them in a sexual manner.
To address the issue of sexual harassment, colleges in recent years have developed extensive policies and programs to educate students about what behaviors constitute sexual harassment (Stanford University, 2006; University of California, 2006). Yet, there is nothing in the literature concerning student beliefs about where on the body it is acceptable to touch and be touched by other students in casual (not intimate) social interactions. What are the normative beliefs for casual social touching on college campuses? Are there different beliefs, depending on the campus, gender, or other student demographics? Do students know where and under what situation it is acceptable to touch others? This study characterizes student beliefs about touching in casual social interactions by mapping touch zones on the body by gender and direction of touch.

\section{Literature on Social Touching}

Before reviewing the literature, there are several issues that need to be addressed to clarify how the present study is different from previous literature on social touching. First, social touch is usually bi-directional and not unidirectional. 
Second, the classification systems used to differentiate touching on various body parts have either been too cumbersome for use in health promotion programs (Hutchinson and Davidson, 1990; Jourard, 1966; Nguyen, Heslin, and Nguyen, 1975; Rosenfeld, Kartus, and Ray, 1976) or the method of categorizing touch by body areas were not well defined (Willis and Rinck, 1983). Third, most touch studies have involved field observations of dyads (DiBiase and Gunnoe, 2004; Guerrero \& Andersen, 1991; Hall and Veccia, 1990; McDaniel \& Andersen, 1998; Regan, Jerry, Narvaez, Johnson, 1999), including studies of athletes (Kneidinger, Maple, Tross, 2001). Other studies are based on selfreports of actual touching by more intimate interactions (family members, partners, or close personal friends), and not touching in casual social interactions. The type of touching that occurs in casual social interactions are not the same as those in more intimate interactions. Fourth, although constructs such as body accessibility (Jourard, 1966) are useful in sexual harassment prevention programming, it does not provide a broader theoretical framework to explain why some touches may be misunderstood as sexually harassing. The studies below are discussed within the context of these four issues.

\section{Observed Social Touching: The Body Accessibility Construct}

Some of the earliest works on body mapping of interpersonal touch was done by Jourard (1966), who investigated how college students were touched on their bodies by their parents and close friends in the previous twelve months. Students were asked to identify where their parents and close same- and opposite-gender friends had touched them in 14 different body "regions:" head, face, neck, shoulder, upper arm, lower arm, hand, chest, stomach, hip, thigh, knee, calf, and foot. The resulting touch patterns reflected varying degrees of body accessibility as a proxy for self-disclosure, depending on the nature of the relationship. Jourard (1966) found that most touching occurred between close opposite-gender friends in the upper torso region. Jourard's model addressed actual social touching between family members and close personal friends, and where one direction of touch (being touched by others) was investigated. There have been numerous studies on body accessibility (Lomranz \& Shapira, 1974; Pedersen, 1973; Willis \& Rinck, 1983), but only two studies focus on the changing nature of body accessibility. Jourard's original study was replicated by Rosenfeld et al. (1976) and Hutchinson and Davidson (1990) to demonstrate that body accessibility is mediated by culture and time. Thus, the degree of body accessibility in 1966 may change decades later, depending on cultural factors.

Rosenfeld et al. (1976) and Hutchinson and Davidson (1990), for example, believed that events such as the "sexual revolution" of the 1960's and 70's, homophobia, AIDS hysteria and emerging concerns about sexual harassment may have resulted in the shifting of cultural beliefs. This shifting in cultural beliefs in turn may have contributed to altered social touch behavior. Rosenfeld et al. (1976) found that males were touched more frequently in their study than Jourard's by close female friends in the chest, stomach, and hip region. Females were touched more frequently by close male friends in the entire torso region. The knees, legs, and feet were not frequently touched. Rosenfeld et al. (1976) also noted that the head, face, arms, and hands were not touched as much as other body regions. They found no significant difference in touch patterns for same-gender friends, but did find opposite-gender friends touched each other more frequently than a decade prior. Hutchinson and Davidson in the 1990s, however, noted decreases in touches between the research participants and their parents and partners.

The body accessibility construct is useful to explain why individuals are touched by others, and studies have demonstrated the influence of culture and time on the construct, but the construct is unidirectional, and is based on recollections of actual touching in intimate relationships (e.g., family members, intimate friends). A bi-directional construct focusing on touch beliefs in casual social interactions may be more useful for health education programming on college campuses. 


\section{Focusing on Touch Beliefs and Touch Zones}

Tomita, Schneit, and Shapiro (2000) continued the body accessibility research from the 1990s, in which they conducted a study in New York City with a multicultural population of students at Brooklyn College. The students completed a Touch Survey consisting of two identical subscales with 35 body parts on each. The subscales differed only in the question being asked of the student ("Where is it OK to touch a male friend?" and "Where is it OK to touch a female friend?"). Rather than asking college students where they were touched in the past year by family members and close personal friends, as was the case in the Jourard (1966) line of research, Tomita and colleagues asked college students where they believed it is acceptable to touch other students in casual relationships. This shifting of the focus on touching others rather than being touched by others added a layer of information that may be useful at some point to understand beliefs about the body accessibility of others rather than of self.

There were several differences in the Tomita et al. (2000) study when compared to previous research on body accessibility: (1) college students were asked about their beliefs rather than recollections of actual touching, (2) college students were asked about touching in casual rather than more intimate social interactions (e.g., family members and close personal friends), and (3) college students were asked about touching others rather than being touched by others.

Tomita et al. (2000) developed the concept of Touch Zones (Public, Discretionary, Private) to reflect student beliefs about the level of "touch acceptability" of body parts. The touch zones were variants of the 11-14 disarticulated body regions from the Jourard (1966) line of research. The touch zones were mapped on the body and color coded as a traffic light: Public (green), Discretionary (yellow), and Private (red). The touch zone concept consisting of three zones was far more useful and realistic for use by college health educators than the 11-14 body regions. Also, use of the color mapping scheme resembling a traffic light was readily understood by college students as Go, Yield/Use Caution, and Stop, although Go does not imply that those body parts in this grouping may be touched in all social situations. This method of grouping body parts into touch zones and using easily recognizable traffic light colors solved the problem of having a sensible system for educating college students about social interactions and preventing situations where their touch may be misunderstood as sexually harassing.

The results of the study revealed that touch zones varied according to the gender of both parties. Same-gender touch beliefs differed markedly between males and females. Male student beliefs about touching other males resulted in the largest private touch zone with the smallest discretionary touch zone (Public $17 \%$, Discretionary 6\%, Private $71 \%$ ). Tomita and colleagues suggested that the small male/male discretionary touch zone indicated well-defined and more rigid cultural beliefs about where males should touch other males. In other words, males, regardless of culture, know where it is acceptable and unacceptable to touch other males, and that there are very few shades of gray. The female/female touch beliefs were more evenly distributed than male/male (Public $29 \%$, Discretionary $26 \%$, Private $46 \%$ ), but the large private touch zone was an unexpected finding since women traditionally are believed to use more touch when interacting socially.

Opposite-gender touch beliefs also differed markedly. Male student beliefs about touching females (Public 11\%, Discretionary 69\%, Private 20\%) revealed a large discretionary touch zone (belief that the body part may be touched under certain circumstances). Tomita et al. (2000) speculated that the large male/female discretionary touch zone may indicate a situation where there may be misunderstandings if, based on culture, a woman may not agree that the body parts are touchable, even in certain situations. An alternate interpretation of the touch zone findings is that men are cautious about touching women in casual relationships because their touches may be misinterpreted as sexually harassing behavior. Note that the public touch zone is very small (11\%), indicating that men 
considered very few body parts to be public, and the discretionary touch zone is very large, indicating that two thirds of body parts are believed to be touchable only in certain circumstances and with caution. The female/male beliefs (Public 23\%, Discretionary $40 \%$, Private 37\%) differed from the male/female beliefs where the touch zones were more evenly distributed. The public touch zone was more than twice the size of the male/female public touch zone.

Tomita et al. (2000) revealed clear gender differences in touch beliefs, but the study was limited in that only one direction of touch was measured. In social interactions, it is important to understand the process of reciprocation, or an individual's beliefs about touching others and being touched by others. The results of the study showed some promise as a supplemental model for use in sexual harassment prevention programming on college campuses.

\section{Exploring Touch Zones}

In 2001, a larger study was conducted by Tomita and colleagues, and the results were reported by Shapiro (2003). The Touch Survey was modified from the previous study to include four, 36-item subscales of body parts plus a demographics page. Two of the 36-item subscales assessed student beliefs about where it is acceptable to touch others in casual social interactions ("Where is it OK to touch a male friend?" and "Where is it OK to touch a female friend?"). The other two 36-item subscales assessedstudent beliefs about where it is acceptable for others to touch them ("Where is it OK for a male friend to touch you?" and "Where is it OK for a female friend to touch you?").

Shapiro (2003) used cluster analyses as in the previous study to examine whether a threecluster solution produced meaningful data. The three-cluster solution (Public, Discretionary, and Private Touch Zones) was essential to examine whether there were significant differences for "touch acceptability" between groups for touch zones, touch direction, gender, and race/ethnicity. Without first grouping the data by touch zone, the results were not significantly different between groups for touch direction, gender, and race/ethnicity. Using ANOVA, Shapiro found no significant differences for touch acceptability according to gender and touch direction, except for female beliefs about males touching them in the private touch zone. Females had a significantly larger private touch zone for males touching them than females touching males.

There were several limitations to Shapiro's (2003) study. First, the final sample size was too small to do an adequate analysis of touch zones by race/ethnicity (a proxy for culture). After parsing the data for marital status and then race/ethnicity, the cells were too small for meaningful analysis. Second, the use of ANOVA to establish differences between groups required the formation of global touch zone memberships to test differences between groups. This analytic approach would have been appropriate for Likert-like scales where subscales have defined memberships, but touch zone memberships change, unlike Likert-like scales. Such changes in membership required statistical methods other than ANOVA to account for the changes. Third, Shapiro did not describe what body parts belonged to what touch zones by gender and direction of touch; thus, percent of body parts for each touch zone were not available for male beliefs about touching and being touched by females, as well as female beliefs about touching and being touched by males.

\section{Summary of Previous Research}

The original line of body accessibility research by Jourard (1966) provided a basis for physical touch and self-disclosure in more intimate, but not sexual, relationships. The body mapping schemata developed from this line of research were cumbersome, and were representative of actual touches in one direction of communication between two people. Later researchers (Hutchinson and Davidson, 1990; Rosenfeld et al., 1976) provided evidence that culture plays a role in mediating the body accessibility construct, thus altering social touch behavior over time and place.

Tomita et al. (2000) changed the focus of Jourard's line of research by shifting the 
emphasis from: 1) actual recollected touches to touch beliefs, 2) touch in casual rather than more intimate social interactions, 3) unidirectional actual touch to bidirectional touch beliefs, and 4) 11-14 disarticulated body regions representing actual touches to three touch zones based on bidirectional touch beliefs (public, discretionary, and private). Studies indicate gender differences in touch beliefs with resulting touch zone patterns illustrating these belief differences. The touch zone patterns have been used for qualitatively examining touch beliefs.

The development of the touch beliefs construct (Tomita et al., 2000) required the development and testing of the Touch Survey that would provide meaningful clustering of body parts into touch zones. Both qualitative and quantitative analytic methods are still needed to analyze these touch zones for between-group differences.

\section{Need for Standardized Method of Analysis}

Although the results from previous studies have been helpful to validate the touch zones and to establish differences for gender and direction of touch, a standardized method of analysis is still needed to compare the touch zones by gender and direction of touch. This line of research will be useful to develop an instrument for use in the classroom whereby students will assess their own beliefs, and discuss the results in group processes to develop insight into the way they non-verbally communicate using touch. Thus, the purpose of this study is to explore the concept of reciprocal touch zones, and to develop a standardized quantitative method to compare touch zones by gender and direction of touch. It is anticipated that the study results will provide further evidence of touch zones (Public, Discretionary, Private), and that there will be gender differences in touch zone patterns.

\section{Methods}

\section{Definitions}

Touch zone refers to a cluster of body parts that are believed to be acceptable to touch in a casual interaction (Public), sometimes, depending on the situation (Discretionary), and only in intimate situations (Private).
Touch zone ratio refers to a standardized number for beliefs about touching others divided by a standardized number for beliefs about being touched by others. The percent of body parts for a touch zone is divided the percent of body parts for a corresponding touch zone. A touch zone ratio of 1.0 with identical body parts is considered reciprocal. For example, the touch zone ratio is computed by dividing the percent the public touch zone for You Touch Male Friend public touch zone (33\%) by the percent for the public touch zone Male Friend Touch You (33\%). The ratio is 1.0 .

Reciprocal touch beliefs refers to the belief that touch zones are mutually corresponding, regardless of the direction of touch, but may differ according to gender and other factors.

Reciprocal touch zones refers to identical clusters of body parts based on individual touch beliefs, and organized into three clusters (Public, Discretionary, Private) representing touch zones. The touch zone ratio for reciprocal touch zones is 1.0 .

\section{Instrument Development}

The Touch Survey is a 144-item, paper-andpencil survey. It contains four identical 36-item Likert-type subscales, differing only in the question being asked of the student: "Where is it OK to touch a male friend?"; "Where is it OK to touch a female friend?"; Where is it OK for a male friend to touch you?"; and "Where is it OK for a female friend to touch you?" The response options are identical for each subscale areNever, Almost Never, Sometimes, Almost Always, and Always. Each subscale lists 36 body parts (cheeks, lips, nose, chin, eyes, ears, forehead, top of head, back of head, hair, back of neck, shoulder, upper back, lower back, buttocks, back of thigh, back of calf, ankle, food, upper arm, elbow, lower arm, wrist, palm of hand, back of hand, fingers, front of neck, chest/breast, abdomen (stomach), flanks (sides), pubic area, genitals, front of thigh, inner thigh, knee cap, shin). Other questions on the survey relate to demographics such as gender, age, marital status, race/ethnicity, religion, sexual orientation, and country of birth. 
Two of the subscales measure beliefs about touching others (You Touch Male Friend and You Touch Female Friend) and two subscales measure beliefs about being touched by others (Male Friend Touch You and Female Friend Touch You). With two directions of touch beliefs, analyses could be done to see if the touch zones (Public, Discretionary, Private) were reciprocal, that is, touch zones are symmetrical where the same body parts for touching others are the same for being touched by others ("If I touch you there, you can touch me in the same place."). Reciprocal touch zones refers to identical clusters of body parts based on individual touch beliefs, and are organized into three clusters (Public, Discretionary, Private) representing touch zones.

If there are reciprocal touch zones, there should be no differences in touch zone patterns for each of the subscales. In other words, the touch zones clustered from each of the subscales should be identical. If there is a difference between subscales, then touch may be an asymmetrical belief, depending on gender, the direction of touch, and other mediating variables that are proxies for culture.

\section{Psychometric Analysis}

Although the Touch Survey is not intended to be used as a summative score scale, Cronbach's alpha was computed for internal consistency reliability from the residential university (RU) $(\mathrm{N}=242)$ and community college $(\mathrm{CC})(\mathrm{N}=$ 200) samples: Touch Survey (RU $\alpha=.990, \mathrm{CC}$ $\alpha=.992$ ), and subscales You Touch Male Friend (RU $\alpha=.969, \mathrm{CC} \alpha=.985$ ), You Touch Female Friend (RU $\alpha=.970, \mathrm{CC} \alpha=.986$ ), Male Friend Touch You (RU $\alpha=.974, \mathrm{CC} \alpha=.986$ ), and Female Friend Touch You (RU $\alpha=.979, \mathrm{CC} \alpha=$ .987).

Each of the Touch Survey subscales were factor analyzed separately using principal components analysis with varimax rotation. Only three factors loaded on each of the subscales, and each of the factors had eigenvalues of 1.0 or greater: You Touch Male Friend (KMO = .957, Bartlett's $\mathrm{p}<.001$, percent variance explained $=$ $79 \%)$, You Touch Female Friend $(\mathrm{KMO}=.956$, Bartlett's $\mathrm{p}<.001$, percent variance explained $=$
$80 \%)$, Male Friend Touch You $(\mathrm{KMO}=.961$, Bartlett's $\mathrm{p}<.001$, percent variance explained $=$ $80 \%)$, Female Friend Touch You (KMO = .958, Bartlett's $\mathrm{p}<.001$, percent variance explained $=$ $82 \%$ ). The factor analytic results reinforce the hierarchical cluster analytic method used in this study where the factors correspond to the three touch zones (Public, Discretionary, Private).

\section{Procedure and Sample Demographics}

This cross-sectional survey study was conducted in 2006/2007 at a residential, public university and a public community college in Northern California. A convenience sample of research participants were solicited through classroom visitation. Data were also collected from a table set up on the campus of the residential university.

The demographic characteristics of both samples were characterized for campus (residential university and community college), gender, age (mean and standard deviation), sexual orientation, race/ethnicity, birth country of parents, birth country of research participant, marital status, currently involved in a relationship, currently have children, and religion.

The residential university sample consisted of 242 white students (147 females and 95 males) with a mean (and standard deviation) age of $21.02 \pm 2.05$, and $100 \%$ heterosexual. This homogenous sample serves as a comparison group for future studies examining for differences in touch beliefs. Nearly all (95.5\%) of their parents and $99.2 \%$ of the participants were born in the USA. Most (95.9\%) were never married, $96.3 \%$ had no children, and 50\% were currently in a relationship. The majority $(89.6 \%)$ of participants had either no religion $(35.5 \%)$ or identified with Christianity (54.1\%).

The community college sample consisted of 200 participants (99 females and 101 males) with a mean age of $21.61 \quad(\mathrm{SD}=4.73)$. The racial/ethnic mix included $68 \%$ White, $10 \%$ Latino, 5\% Asian/Pacific Islander, 4\% African American, and $13 \%$ other groups. Also, $92.5 \%$ of participants were heterosexual, and $78.5 \%$ of their parents and $85.5 \%$ of the participants 
themselves were born in the USA. Most (86.5\%) were never married, $86.5 \%$ had no children, and $45.5 \%$ were currently in a relationship. The majority $(81.5 \%)$ of participants had either no religion $(31.5 \%)$ or identified with Christianity $(50.0 \%)$.

The study procedures were explained to the participants on both campuses, and they were asked to complete a voluntary paper-and-pencil survey. The approximate time for completion was 10-15 minutes. This study was approved by the respective institutional review boards.

\section{Data Analysis}

Statistical Package for the Social Sciences ${ }^{\circledR}$ (SPSS) 15.0 was used to analyze the data. The data were analyzed using hierarchical cluster analyses with a between-groups linkages method and squared Euclidean distances measure. A three-cluster solution (Shapiro, 2003; Tomita et al., 2000) was selected for the development of Touch Zones (Public, Discretionary, and Private), which has allowed for meaningful interpretation of the data in past studies. For validation of the clustering method, see Shapiro (2003) and the factor analytic data presented above. The dataset was analyzed by campus (residential university and community college), gender, and direction of touch. Thedataset was analyzed by campus because of the unique but related communities where health educators are employed. Each campus community is presumed to have a different student culture, with different social interactions (e.g., residential university students live together away from home and community college students commute from home), thus, the campus setting may be a proxy for culture, and differences in touch beliefs may be revealed.

Percentages were generated according to the percent of body parts in each of the clusters (touch zones). Touch zone ratios were computed dividing the touch zone percentages for touching others by the touch zone percentages for being touched by others. The percentages for You Touch Male Friend or You Touch Female Friend were divided by Male Friend Touch You or Female Friend Touch You. A ratio of 1.0 with identical body parts in each touch zone were considered reciprocal.

The higher the ratio ( $>1.0)$, the greater the touch zone imbalance between touching others and being touched by others, where beliefs about touching others exceed that of being touched by others. For the public and discretionary touch zones, the research participant believes many more body parts on others are acceptable to touch than what others may touch on the participant's body. For the private touch zone, a high ratio indicates the research participant believes many more body parts on others are "off-limits" for him/her to touch than what the participant believes is acceptable for others to touch him/her.

The lower the ratio $(<1.0)$, the greater the touch zone imbalance between being touched by others, where beliefs about being touched by others exceed that of touching others. For the public and discretionary touch zone ratios, the research participant believes fewer body parts on others are acceptable to touch than what others may touch on his/her own body. For the private touch zone, a low ratio indicates the research participant believes many more body parts on his/her own body are "off-limits" to touch by others than what the participant believes is acceptable for him/her to touch others.

The analytic methods used in this study appear to have produced meaningful results that may be useful to college health educators. As in previous studies (Shapiro, 2003; Tomita et al., 2000), hierarchical cluster analyses using a three-solution method continues to be useful in translating touch beliefs into touch zones. The psychometric data presented appear to confirm that the instrument is reliable and the touch zones appropriately divided into three groups.

Computing percent of body parts for each touch zone was useful to describe quantitatively the relative size of the touch zones. The percentages, however, did not accurately describe the qualitative characteristics of proportion and shape. Thus, Appendix A and Appendix B mapped the touch zones to provide qualitative data. The zones were colored coded as a traffic light by the principal investigator: Green 
(Public), Yellow (Discretionary), and Red (Private), but the Green color of the public touch zone does not imply that the zone may be touched in all social situations.

In addition to characterizing the touch zones, touch zone ratios were computed as a preliminary step for the development of an instrument for use in the classroom. The use of ratios to illustrate the relationship between touching others and being touched by others was useful to visualize the reciprocal nature of touch in each zone. The concept of a 1.0 touch zone ratio may be easily understood by students, where variance from the 1.0 ratio would indicate the lack of reciprocal touch, and class discussions could then revolve around touch zone norms in their college communities.

The touch zone ratios were deemed sufficient to express the differences between touch zones in this study without having to use more advanced analytic methods. Other types of statistical methods were viewed as unnecessary because the cluster analyses were sufficiently robust to establish touch zones, and touch zone ratios standardized the touch zone relationships.

\section{Results}

\section{Global Touch Zones}

Hierarchical cluster analyses using a threesolution method (Shapiro, 2003; Tomita et al., 2000) were used to cluster the body parts into touch zones across all four subscales, regardless of touch direction and gender.

The Public Touch Zone membership was larger for residential university students than community college students. The Public Touch Zone membership (24 body parts) for the residential university students were: ankle, back of calf, back of hand, back of head, back of neck, cheeks, chin, ears, elbow, fingers, foot, forehead, hair, knee cap, lower arm, lower back, nose, palm of hand, shin, shoulder, top of head, upper back, upper arm, wrist. The Public Touch Zone membership (13 body parts) for the community college students were: back of hand, back of head, elbow, fingers, hair, lower arm, palm of hand, shoulder, top of head, upper arm, upper back, wrist. The body parts that both samples had in common were: back of hand, back of head, elbow, fingers, hair, lower arm, palm of hand, shoulder, top of head, upper back, upper arm, and wrist.

The Discretionary Touch Zone membership (nine body parts) for the residential university students were: abdomen (stomach), back of thigh, buttocks, chest/breast, eyes, flanks (sides), front of neck, front of thigh, lips. The Discretionary Touch Zone membership (21 body parts) for the community college students were: abdomen, ankle, back of calf, back of neck, back of thigh, buttocks, cheeks, chest/breast, chin, ears, eyes, flanks, foot, forehead, front of neck, front of thigh, knee, lips, lower back, nose, shin. The body parts that both samples had in common were: lips, eyes, buttocks, back of thigh, front of neck, chest/breast, abdomen (stomach), and front of thigh)

The Private Touch Zone membership (three body parts) for the residential university students were: genitals, inner thigh, pubic area. The Private Touch Zone membership (21 body parts) for the community college students were: genitals, inner thigh, pubic area. The global Private Touch Zone body parts were identical for both residential university and community college students.

\section{Touch Zones by Gender and Touch Direction}

A summary of the hierarchical cluster analyses of touch zones by gender and direction of touch is presented in Table 1, and illustrated in Appendix A and Appendix B. Percentage of body parts are reported according to gender, touch zone (public, discretionary, private), and touch survey subscale (You Touch Male Friend, You Touch Female Friend, Male Friend Touch You, and Female Friend Touch You). The results presented below are divided into samegender and opposite-gender results. For each of these subsections, the characteristics of the three touch zones are discussed by direction of touch. 
Table 1

Percent of body parts by Touch Survey subscale, gender, institution [Residential University (RU) and Community College (CC)], and touch zone.*

\begin{tabular}{|c|c|c|c|}
\hline & \multicolumn{3}{|c|}{ Percent of Body Parts by Touch Zone } \\
\hline $\begin{array}{c}\text { Touch Survey Subscales by Gender } \\
\text { and Direction of Touch }\end{array}$ & $\begin{array}{c}\text { Public } \\
\text { RU/CC }\end{array}$ & $\begin{array}{c}\text { Discretionary } \\
\text { RU/CC }\end{array}$ & $\begin{array}{c}\text { Private } \\
\text { RU/CC }\end{array}$ \\
\hline Male Research Participants (RU N=95, CC N=101) & & & \\
\hline You Touch Male Friend (YTMF) & $33 / 8.3$ & $44 / 36.1$ & $22 / 55.6$ \\
\hline You Touch Female Friend (YTFF) & $39 / 36.1$ & $47 / 47.2$ & $14 / 16.7$ \\
\hline Male Friend Touch You (MFTY) & $33 / 25$ & $56 / 63.9$ & $11 / 11.1$ \\
\hline Female Friend Touch You (FFTY) & $42 / 38.9$ & $47 / 55.6$ & $11 / 5.6$ \\
\hline \multicolumn{2}{|c|}{} & & \\
\hline Female Research Participants (RU N=147, CC N=99) & & & \\
\hline You Touch Male Friend (YTMF) & $64 / 36.1$ & $28 / 30.6$ & $8 / 33.3$ \\
\hline You Touch Female Friend (YTFF) & $64 / 66.7$ & $25 / 16.7$ & $11 / 16.7$ \\
\hline Male Friend Touch You (MFTY) & $36 / 61.1$ & $33 / 27.8$ & $31 / 11.1$ \\
\hline Female Friend Touch You (FFTY) & $67 / 52.7$ & $22 / 41.7$ & $11 / 5.6$ \\
\hline
\end{tabular}

*Row percentages not totaling to $100 \%$ are due to rounding errors.

\section{Same-Gender Touch Zones}

Male research participant beliefs about where it is acceptable to touch other male students and where it is acceptable to be touched by other male students were analyzed. This combination of touch zones will be referred to as "male/male." The ratios for the public, discretionary, and private touch zones were Jshaped, with increased caution in the private touch zone by male research participants for touching other males (see Table 1, Appendix A, Appendix C).

\section{Residential University Male/Male}

The male/male public touch zones were identical: back of hand, back of head, elbow, fingers, hair, lower arm, palm of hand, shoulder, top of head, upper arm, upper back, wrist (see Table 1 and Appendix C (Figure 1a). A male/male public touch zone ratio was computed by dividing the percent of body parts according to direction of touch (You Touch Male Friend divided by Male Friend Touch You). The ratio was 1.0 with identical body parts. A touch zone ratio of 1.0 with identical body parts is considered to be reciprocal.
The male/male discretionary touch zone was smaller for You Touch Male Friend (44\% of body parts) [abdomen, ankle, back of calf, back of neck, cheeks, chest/breast, chin, ears, flanks, forehead, foot, front of neck, knee cap, lower back, nose, shin], than Male Friend Touch You (56\% of body parts) [abdomen, ankle, back of calf, back of neck, back of thigh, buttocks, cheeks, chest/breast, chin, ears, eyes, flanks, forehead, foot, front of neck, knee cap, lips, lower back, nose, shin]. The five body parts that differed were lips, eyes, buttocks, lower back, and back of thigh. The discretionary touch zone ratio was .79.

The male/male private touch zone was twice the size for You Touch Male Friend (22\%) than Male Friend Touch You (11\%), indicating male research participants were more cautious about touching body parts that could be considered private. The identical body parts were front of thigh, genitals, inner thigh, and pubic area. You Touch Male Friend included the additional body parts of buttocks, back of thigh, eyes, and lips. The private touch zone ratio was 2.0. 


\section{Community College Male/Male}

The male/male public touch zones were not identical as they were with residential university male participants (see Table 1). The public touch zone was three times smaller for You Touch Male Friend (8.3\%) (back of hand, fingers, palm of hand) than Male Friend Touch You (25\%) (back of hand, elbow, fingers, lower arm, palm of hand, shoulder, upper back, upper arm, wrist) (see Table 1 and Appendix C, Figure 1b). A male/male public touch zone ratio was computed by dividing the percent of body parts according to direction of touch (You Touch Male Friend divided by Male Friend Touch You). The public touch zone ratio was 0.33 , lower than the reciprocal touch zone for residential university males (1.0).

The male/male discretionary touch zone was smaller for You Touch Male Friend $(36.1 \%)$ [back of head, back of neck, elbow, hair, knee cap, lower arm, lower back, shin, shoulder, top of head, upper arm, upper back, wrist], than Male Friend Touch You (63.9\%) [abdomen, ankle, back of head, back of calf, back of neck, back of thigh, cheeks, chest/breast, chin, ears, eyes, flanks, foot, forehead, front of neck, front of thigh, hair, knee cap, lips, lower back, nose, shin, top of head]. The discretionary touch zone ratio was .56 , lower than residential university males (.79).

The male/male private touch zone was five times smaller for You Touch Male Friend (55.6\%) (abdomen, ankle, back of calf, buttocks, back of thigh, cheeks, chest/breast, chin, eyes, ears, flanks (sides), foot, forehead, front of neck, front of thigh, genitals, inner thigh, lips, nose, pubic area) than Male Friend Touch You (11.1\%) (buttocks, genitals, inner thigh, pubic area), indicating male research participants were cautious about touching body parts that could be considered private. The private touch zone ratio was 5.0, two and a half times the ratio for residential university males (2.0).

\section{Residential University Female/Female}

Female research participant beliefs about where it is acceptable to touch other female students and where it is acceptable to be touched by other female students were analyzed. This combination of touch zones will be referred to as "female/female." The female/female touch zone patterns hovered around 1.0 for all three touch zones, and while not technically reciprocal in nature, the results came pretty close to being reciprocal.

The female/female public touch zones differed by only one body part (64\% You Touch Female Friend and 67\% Female Friend Touch You). The identical body parts were: ankle, back of hand, back of head, back of neck, cheeks, chin, ears, elbow, fingers, foot, forehead, hair, knee cap, lower arm, lower back, nose, palm of hand, shin, shoulder, top of head, upper arm, upper back, and wrist. Female Friend Touch You included the additional body part of back of calf. The public touch zone ratio was 96 .

The female/female discretionary touch zones differed by only one body part (25\% You Touch Female Friend and 22\% Female Friend Touch You). The identical body parts were: abdomen, back of calf, back of thigh, buttocks, eyes, flanks, front of neck, front of thigh, and lips. You Touch Female Friend included the additional body part of back of calf. The discretionary touch zone ratio was 1.14.

The female/female private touch zones were identical in body parts: chest/breast, genitals, inner thigh, andpubic area; thus, the female/female private touch zone ratio was 1.0. A touch zone ratio of 1.0 with identical body parts is considered to be reciprocal.

\section{Community College Female/Female}

Female research participant beliefs about where it is acceptable to touch other female students and where it is acceptable to be touched by other female students were analyzed. This combination of touch zones will be referred to as "female/female." While the residential university female/female touch zone patterns hovered around 1.0 for all three touch zones, the community college female/female touch zones did not.

The female/female public touch zone was larger for You Touch Female Friend $(66.7 \%)$ than Female Friend Touch You (52.7\%). The 
identical body parts were: back of hand, back of head, back of neck, cheeks, chin, elbow, ears, fingers, forehead, hair, lower arm, lower back, nose, palm of hand, shoulder, top of head, upper arm, upper back, and wrist. There were five additional body parts for You Touch Female Friend: ankle, eyes, foot, knee cap, and shin. The public touch zone ratio was 1.27 , compared to residential university .96 .

The female/female discretionary touch zone was two and a half times smaller for You Touch Female Friend (16.7\%) (abdomen, back of calf, flanks, front of neck, front of thigh, lips) than Female Friend Touch You (41.7\%) (abdomen, ankle, back of calf, back of thigh, buttocks, chest/breast, eyes, flanks, foot, front of neck, front of thigh, inner thigh, knee cap, lips, shin). The discretionary touch zone ratio was .40, compared to residential university 1.14 .

The female/female private touch zone was three times larger for You Touch Female Friend (16.7\%) (back of thigh, buttocks, chest/breast, genitals, inner thigh, pubic area) than Female Friend Touch You (5.6\%) (genitals, pubic area). The private touch zone ratio was 2.98, compared with residential university 1.0 .

\section{Opposite-Gender Touch Zones}

\section{Residential University Male/Female}

Male research participant beliefs about where it is acceptable to touch female students and where it is acceptable to be touched by female students were analyzed. This combination of touch zones will be referred to as "male/female." The ratios for the public, discretionary, and private touch zones were J-shaped on a graph, with increased caution in the private touch zone by male research participants for touching females (see Appendix B, Appendix C).

The male/female public touch zones $(39 \%$ You Touch Female Friend, 42\% Female Friend Touch You), included the identical body parts: back of hand, back of head, back of neck, elbow, fingers, hair, lower arm, lower back, palm of hand, shoulder, top of head, upper arm, upper back, and wrist. Female Friend Touch You included the additional body part of forehead.
The male/female public touch zone ratio was 93.

Male/female discretionary touch zones were symmetrical, but not reciprocal, with both touch zones containing $47 \%$ of body parts. The You Touch Female Friend and Female Friend Touch You discretionary touch zones were identical for: abdomen, ankle, back of calf, back of thigh, buttocks, cheeks, chin, ears, eyes, flanks, foot, knee cap, lips, nose, and shin. The You Touch Female Friend discretionary touch zone included the additional body part of forehead and the Female Friend Touch You included the additional body part of front of neck. Even though the touch zone ratio was 1.0, the body parts are not identical, thus, the discretionary touch zones are not reciprocal.

The male/female private touch zones differed by only one body part (14\% You Touch Female Friend, 11\% Female Friend Touch You). The identical body parts were: front of thigh, genitals, inner thigh, and pubic area. You Touch Female Friend included the additional body part of chest/breast. The male/female private touch zone ratio was 1.27 .

\section{Residential University Female/Male}

The female/male public touch zones were greatly disproportionate (64\% You Touch Male Friend, 36\% Male Friend Touch You). The identical body parts were: back of hand, back of head, fingers, lower arm, lower back, palm of hand, shoulder, top of head, upper back, wrist. You Touch Male Friend included the additional body parts of ankle, back of neck, cheeks, chin, ears, elbow, forehead, foot, knee cap, nose, and shin. The female/male public touch zone ratio was 1.78 .

The female/male discretionary touch zones were similar (28\% You Touch Male Friend, 33\% Male Friend Touch You). Only the body part back of calf was identical in both zones. You Touch Male Friend included the additional body parts: abdomen, back of calf, back of thigh, buttocks, chest/breast, eyes, front of neck, lips, flanks, and front of thigh. Male Friend Touch You included the additional body parts: ankle, back of neck, cheeks, chin, ears, foot, knee cap, 
nose, shin, and forehead. The female/male discretionary touch zone ratio was .85 .

The female/male private touch zones were greatly disproportionate (8\% You Touch Male Friend, 31\% Male Friend Touch You). The identical body parts were: genitals, inner thigh, and pubic area. Male Friend Touch You included the additional body parts: abdomen, back of thigh, buttocks, chest/breast, eyes, flanks, front of neck, front of thigh, and lips. The female/male public touch zone ratio was .26.

\section{Community College Male/Female}

The male/female public touch zone You Touch Female Friend (36.1\%) (back of hand, back of head, elbow, fingers, hair, lower arm, lower back, palm of hand, shoulder, top of head, upper arm, upper back, wrist) was smaller than Female Friend Touch You (38.9\%) (back of hand, back of head, back of neck, elbow, fingers, hair, lower arm, lower back, palm of hand, shoulder, top of head, upper arm, upper back, wrist). The male/female public touch zone ratio was .93 .

The male/female discretionary touch zone You Touch Female Friend (47.2\%) (abdomen, ankle, back of calf, back of neck, cheeks, chin, ears, eyes, flanks, foot, forehead, front of neck, front of thigh, knee cap, lips, nose, shin) was smaller than Female Friend Touch You (55.6\%) (abdomen, ankle, back of calf, back of thigh, buttocks, cheeks, chest/breast, chin, ears, eyes, flanks, foot, forehead, front of neck, front of thigh, inner thigh, knee cap, lips, nose, shin). The male/female discretionary touch zone ratio was .85.

The male/female private touch zone You Touch Female Friend (16.7\%) (back of thigh, buttocks, chest/breast, genitals, inner thigh, pubic area) was a third the size of Female Friend Touch You (5.6\%) (genitals, public area). The male/female private touch zone ratio was 2.98 .

\section{Community College Female/Male}

The female/male public touch zone You Touch Male Friend (36.1\%) (back of hand, back of head, elbow, fingers, forehead, hair, lower arm, palm of hand, shoulder, top of head, upper arm, upper back, wrist) was smaller than Male Friend
Touch You (61.1\%) (ankle, back of head, back of neck, back of hand, cheeks, chin, ears, elbow, fingers, foot, forehead, hair, knee cap, lower arm, nose, palm of hand, shin, shoulder, top of head, upper arm, upper back, wrist). The female/male public touch zone ratio was .59 .

The female/male discretionary touch zone You Touch Male Friend (30.6\%) (ankle, back of neck, cheeks, chin, ears, foot, knee cap, lower back, nose, shin) was larger than Male Friend Touch You (27.8\%) (lips, eyes, lower back, buttocks, back of thigh, back of calf, front of neck, abdomen, flanks, front of thigh). The female/male discretionary touch zone ratio was 1.10 .

The female/male private touch zone You Touch Male Friend (33.3\%) (abdomen, back of thigh, buttocks, chest/breast, eyes, flanks, front of neck, front of thigh, genitals, inner thigh, lips, pubic area) was three nearly times larger than Male Friend Touch You (11.1\%) (chest/breast, genitals, inner thigh, pubic area). The female/male private touch zone ratio was 2.87 .

\section{Comparing Touch Zone Ratios}

Same-gender touch zone ratios should have been 1.0 if touch zones are reciprocal. The touch zone ratios were different for male/male and female/female touch zones (see Appendix C).

\section{Residential University}

The residential university male/male touch zone ratios were 1.0 Public 1.0, Discretionary .79, and Private 2.0, where there were reciprocal public touch zones. The male/female touch zones were Public .93, Discretionary 1.00, Private 1.27.

The residential university female/female touch zone ratios were Public .96, Discretionary 1.14, and Private 1.0, with reciprocal private touch zones. The female/male touch zone ratios were Public 1.78, Discretionary .85, and Private .26.

While all three female/female touch zone ratios grouped around the 1.0 ratio, the male/male private touch zone ratio doubled that of the male/male public touch zone ratio. 
The female/male public and private touch zone ratios were opposite that of other groups, with the public touch zone ratio much higher than other groups and the private touch zone ratio much lower (see Appendix C Figure 1A).

\section{Community College}

The community college male/male touch zone ratios were Public .33, Discretionary .56, and Private 5.00. The male/female touch zone ratios were Public .93, Discretionary .85, and Private 2.98 .

The community college female/female touch zone ratios were Public 1.27, Discretionary .40, and Private 2.98. The female/male touch zone ratios were Public .59, Discretionary 1.10, and Private 2.87.

\section{Discussion}

The results of this study provide a preliminary view of student touch beliefs and the resulting touch zone patterns, but at this point, the results should be interpreted as exploratory in nature. The touch zone patterns between the New York City (Shapiro, 2003; Tomita et al., 2000) and this California study, for example, were very different, and further studies are needed to examine how culture (or proxies for culture) influences changes in touch zones.

\section{Global Touch Zones}

The global touch zone memberships were different between the residential university and community college samples. The public touch zone in the residential university sample was nearly twice as large as the community college sample. In both samples, however, the three body parts in the private touch zones were identical (genitals, inner thigh, pubic area).

The large differences in the public and discretionary touch zones between campuses may be due to demographic factors other than campus and gender, but extensive analyses were not done in this study on other demographic characteristics because such analyses would have parsed the data into small cells that would not have produced meaningful and reliable statistical results. Future studies isolating one or two of these other demographics for their effects on touch beliefs may produce more meaningful results that are verifiable.

In this study, there were demographic differences, however, between the residential university and community college samples for sexual orientation (RU 100\% heterosexual, CC 92.5\%), race/ethnicity (RU 100\% White, CC $68 \%$ ), birth country of parents (RU $95.5 \%$ born in USA, CC $78.5 \%$ ), birth country of research participant (RU 99.2\% born in USA, CC $85.5 \%$ ), marital status (RU $95.9 \%$ never married, CC 86.5\%), currently involved in a relationship (RU 50\%, CC 45.5\%), currently have children (RU $96.3 \%$ have no children, CC $86.5 \%$ ), and religion (RU 89.6\% either have no religion or identify with Christianity, CC $81.5 \%)$.

Aside from the demographic data collected from the research participants, it is common knowledge that the students attending the residential university tend to come from affluent families residing in urban/suburban areas such as the San Francisco Bay area and Los Angeles, whereas the community college students tend to come from working class families residing in rural areas of Northern California. Future research is needed to focus on student diversity as a factor that may contribute to greater variety of touch zone patterns. With an increase in touch zone patterns, there may be an increased likelihood of miscommunication through touch.

\section{Same-Gender Touch Zones Male/Male}

The residential university male/male public touch zones and the female/female private touch zones were reciprocal, but all others were not (see Appendix A, Appendix C). If all three touch zones were reciprocal, we would have seen a touch zone ratio of 1.0 for each. This leads to the question of why it is acceptable to touch others in body areas but not acceptable to be touched in the same body areas?

The residential male/male public and discretionary touch zone ratios (1.0 and .79, respectively) were reciprocal and near reciprocal, but the private touch zone ratio was 2.0. Likewise, the community college male/male 
public touch zone was .33, the lowest of all male/male ratios, possibly indicating hesitance in touching other males. The private touch zone for community college male/male was 2.0 , the same as the residential university sample. This high private touch ratio may indicate male concerns over having their touch being misinterpreted by another male as being sexual in nature. Previous studies have suggested that same-gender touch between females is more common than between males possibly due to homophobia (Floyd, 2000; Roese, Olson, Borenstein, Martin, Shores, 1992).

A striking difference between the residential university and community college male/male touch zone patterns was the public touch zone. Residential university males believed it was acceptable to touch much of the upper torso of another male whereas community college males believed only the wrist and hands were acceptable to touch. Also, while residential university males believed it was acceptable to be touched in the buttocks by another male under certain circumstances (discretionary touch zone), community college males did not.

\section{Female/Female}

The residential university female/female touch zones were the closest to what would be considered reciprocal: Public .96, Discretionary 1.14, and Private 1.0. The ratios may represent women being more comfortable than men to use touch in same-gender interactions. Residential university females, for example, believed it was acceptable to touch another female's buttocks and be touched in the buttocks by other females under certain circumstances (discretionary touch zone). Community college females, however, did not believe it was acceptable to touch another female's buttocks, but did indicate under certain circumstances (discretionary touch zone) it was acceptable to touch another female in the inner thigh area, and be touched in the same area by another female. Also, although the breasts were consistently categorized in the private touch zone, community college females believed it was acceptable to touch another female's breasts under certain circumstances (discretionary touch zone).

\section{Opposite-Gender Touch Zones}

The touch zone ratio patterns for male beliefs about touching females and females touching them appeared to show caution on the part of the male research participants to touch body parts that could be interpreted as a private touch zone (residential university 1.27, community college 2.98). Further study is needed to clarify this finding. The residential university male/female public (.93) and discretionary (1.0) touch zone ratios indicate nearly reciprocal touch zones. The community college male/female public (.93) and discretionary (.85) touch zone ratios indicate more cautious beliefs about opposite-gender touching. Those touch zone ratios below 1.0 indicate fewer body parts are considered acceptable to touch on a female than what the female is allowed to touch on the male.

A significant finding in this study was the residential university female beliefs about touching males and being touched by males. Female research participants believed it was acceptable to touch nearly twice the number of body parts (ratio 1.78) in the public touch zone on a male than it was acceptable for a male to touch her (Appendix C Figure 1A). In the private touch zone, nearly four times the number of body parts was off limits to males touching her than her touching males (private touch zone ratio .26). This public and private touch zone patterns were contrary to what was found with the community college sample, and from any male/female touch zones (see Appendix C). The community college female/male public touch zone was .59 and private touch zone ratio was 2.87 , indicating more openness to touch in the public touch zone and more guarded touch beliefs about touching men in body parts that could be interpreted as private. The residential university female/male findings will need further study because it was the only opposite-gender finding that went counter to all other groups.

\section{Limitations}

A limitation in this study is the convenience sampling strategy used on both campuses for this study. A non-randomized sample may have been biased because only those students who had access to the research team would have completed the survey. Thus, the results of this 
study are not generalizable to other populations of students. A second limitation is the lack of stability statistics for the Touch Survey instrument that would help interpretation of the findings for this study. Test-retest of the Touch Survey could have been done during this study period. This task will be left to future studies to assure touch beliefs and touch zones are relatively stable.

\section{Implications for Practitioners}

College health educators need current researchbased information about where students believe it is acceptable to touch and be touched in casual social interactions. Such information may be useful as part of a comprehensive sexual harassment prevention program with incoming freshman to sensitize them to the issue, and for the students to discuss the touch research findings for their campus. The touch beliefs line of research is only meant to complement models currently in use at colleges and universities.

It may be useful to conduct future studies with a more diverse sample in collaboration with a health education or the freshman orientation office. It would also be interesting to conduct a parallel study of touch beliefs among students, staff and faculty in the same institution. A broader study with a larger population would provide a snapshot of the "touch culture" in that institution.

This study revealed several interesting findings that may have implications for sexual harassment prevention programming on college campuses. First, there were no opposite-gender reciprocal touch zones. The opposite-gender, Jshaped touch zone ratio patterns (see Appendix C), with the exception of the residential university females, indicated guarded beliefs about touching body parts that may be considered private. Even male students were more hesitant to touch female body parts that may be interpreted as private, contrary to popular beliefs about male students being touchy with females.

The J-shaped pattern may be desirable for a college student population, especially for male beliefs about touching females, since this is where misunderstandings are likely to lead to allegations of sexual harassment. In the case of the residential university females going against all other groups in touch zone patterns, university health educators may want to educate this population about touching male students and not communicating, "I can touch you, but you cannot touch me." Anecdotal evidence suggests that male students may misinterpret female touches as indicators of a more intimate level of relationship than what is reality.

Second, the male same-gender results suggests that the community college students were more than twice as guarded as the residential university male students about touching body parts that may be interpreted as private. The residential university male students were more accepting of being touched by other males than the community college male students. While there is no evidence of homophobia as a cause of this difference between campus male populations, this introduces concerns for college health educators about misunderstandings between residential university males (mostly urban and suburban) and community college (rural) males transferring to those institutions. Would a newly transferred community college male to the residential university misinterpret another male's touch as sexual, even though the touch was not considered, within that university's culture, as being sexual in nature? It would be interesting to examine whether transferring from a community college campus to a residential university campus would have an effect of modifying student beliefs about samegender (and opposite-gender) touch.

Third, there were only two reciprocal touch zones from the residential university sample (male/male public and female/female private). If there are reciprocal touch zones, college health educators are not likely to see misunderstandings in these same-gender social interactions. There were no other reciprocal touch zones by campus, gender, or direction of touch. The residential university sample was very homogeneous, which may explain why there were two reciprocal touch zones. College health educators should not assume that there will be any reciprocal touch zones with their 
student population, and programming should begin with assessing student beliefs. Since touch beliefs may change over time, such reassessment may need to be conducted on a regular basis and integrated into a comprehensive sexual harassment prevention program.

\section{References}

American Association of University Women Educational Foundation. (2001). Hostile hallways. Retrieved February 1, 2008, from http://www.aauw.org/research/upload/hostilehallways.pdf

American Association of University Women Educational Foundation. (2002). Harassment-free hallways: How to stop sexual harassment in Schools. Retrieved February 1, 2008, from http://www.aauw.org/research/upload/completeguide.pdf

Dibiase, R., \& Gunnoe, J. (2004). Gender and culture differences in touching behavior. Journal of Social Psychology, 144, 49-62.

Floyd, K. (2000). Affectionate same-sex touch: The influence of homophobia on observers' perceptions. Journal of Social Psychology, 140, 774-788.

Guerrero, L. K., \& Andersen, P. A. (1991). The waxing and waning of relational intimacy: Touch as a function of relational stage, gender and touch avoidance. Journal of Social and Personal Relationships, 8, 147-165.

Hall, J. A., \& Veccia, E. M. (1990). More 'touching' observations: New insights on men, women, and interpersonal touch. Journal of Personality and Social Psychology, 59, 1155-1162.

Hill, C., and Silva, E. (2006). Drawing the line: Sexual harassment on campus. Retrieved February 26, 2008, from http://www.aauw.org/research/upload/DTLFinal.pdf

Hutchinson, K. L., and Davidson, C. A. (1990). Body accessibility re-revisited: The 60s, 70s and 80s. Journal of Social Behavior and Personality, 5, 341-352.

Jourard, S. M. (1966). An exploratory study of body accessibility. British Journal of Social and Clinical Psychology, 5, 221-131.

Kneidinger, L. M., Maple, T. L., Tross, S. A. (2001). Touching behavior in sport: Functional components, analysis of sex differences, and ethological considerations. Journal of Nonverbal Behavior, 25, 42-62.

Lomranz, J., \& Shapira, A. (1974). Communicative patterns of self-disclosure and touching behavior. Journal of Psychology: Interdisciplinary and Applied, 88, 223-227.

Major, B., Schmidlin, A. M., \& Williams, L. (1990). Gender patterns in social touch: The impact of setting and age. Journal of Personality and Social Psychology, 58, 634-643.

McDaniel, E., \& Andersen, P. A. (1998). International patterns of interpersonal tactile communication: A field study. Journal of Nonverbal Behavior, 22, 59-75.

Nyugen, T., Heslin, R., and Nyugen, M. L. (1975). The meanings of touch: Sex differences. Journal of Communication, 25, 92-103.

Pedersen, D. M. (1973). Self-disclosure, body-accessibility, and personal space. Psychological Reports, 33, 975-980.

Regan, P. C., Jerry, D., Narvaez, M., Johnson, D. (1999). Public displays of affection among Asian and Latino heterosexual couples. Psychological Reports, 84, 1201-1202.

Remland, M. S., Jones, T. S., and Brinkman, H. (1995). Interpersonal distance, body orientation, and touch: Effects of culture, gender, and age. The Journal of Social Psychology, 135, 281-297.

Roese, N. J., Olson, J. M., Borenstein, M. N., Martin, A., \& Shores, A. L. (1992). Same-sex touching behavior: The moderating role of homophobic attitudes. Journal of Nonverbal Behavior, 16, 249259.

Rosenfeld, L. B., Kartus, S., and Ray, C. (1976). Body accessibility revisited. Journal of Communication, 26, 27-30.

Shapiro, R. D. (2003). A study of touch acceptability among college students in casual relationships. Unpublished Doctoral Dissertation, New York University, School of Education.

Stanford University. (2006). Sexual violence awareness, prevention \& support at Stanford. Retrieved January 15, 2008, from http://www.stanford.edu/group/svab/harassment.shtml 
Tomita, M., Schneit, A., Shapiro, R. D. (2000). The use of touch in casual relationships among college students. 4th Annual Faculty Day Conference, Brooklyn College (CUNY), May 26, 2000.

University of California. (2006). University of California policy on sexual harassment. Retrieved January 15, 2008, from http://www.ucop.edu/ucophome/coordrev/policy/PP021006Policy.pdf

Willis, F. N., \& Rinck, C. M. (1983). A personal log method for investigating interpersonal touch. Journal of Psychology: Interdisciplinary and Applied, 113, 119-122.

$\underline{\text { Author Information }}$

Mark Tomita, PhD, CHES

Department of Health and Community Services

California State University, Chico

Chico, CA 95929-0505

Ph.: 530-898-4417

Fax.: 530-898-5107

E-Mail: mtomita@csuchico.edu 


\section{Appendix A}

\section{Same-Gender Touch Zones}

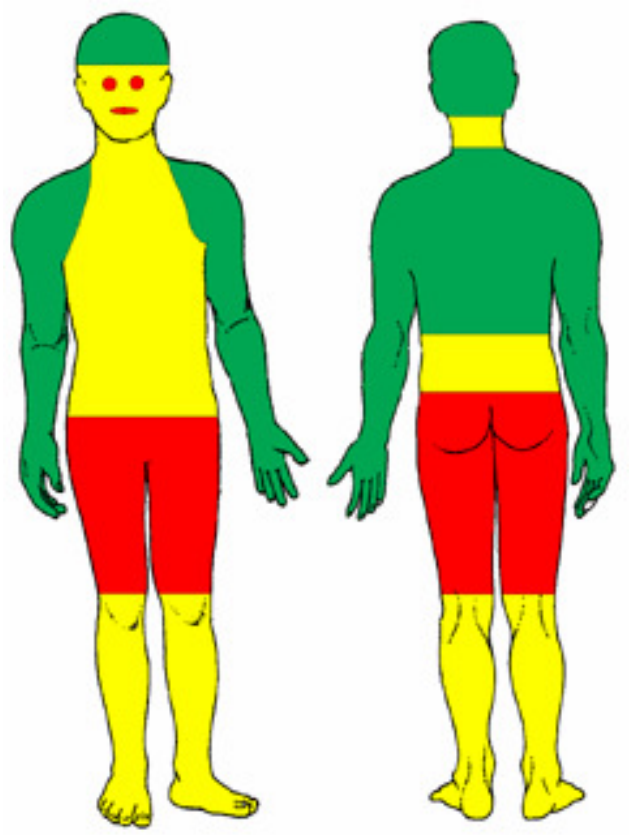

A-1a. Male beliefs about where they may touch other male bodies.

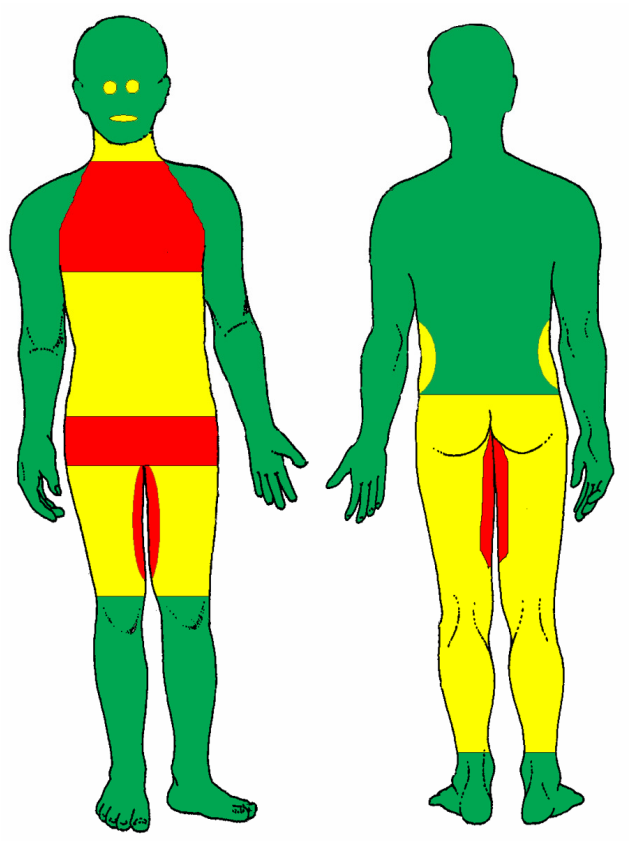

A-1c. Female beliefs about where they may touch other female bodies.

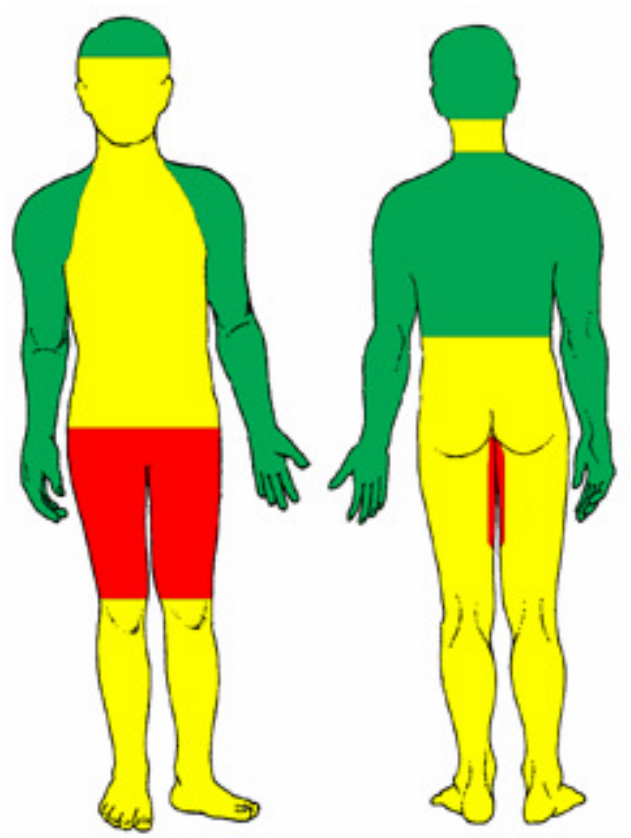

A-1b. Male beliefs about where other males may touch their bodies.

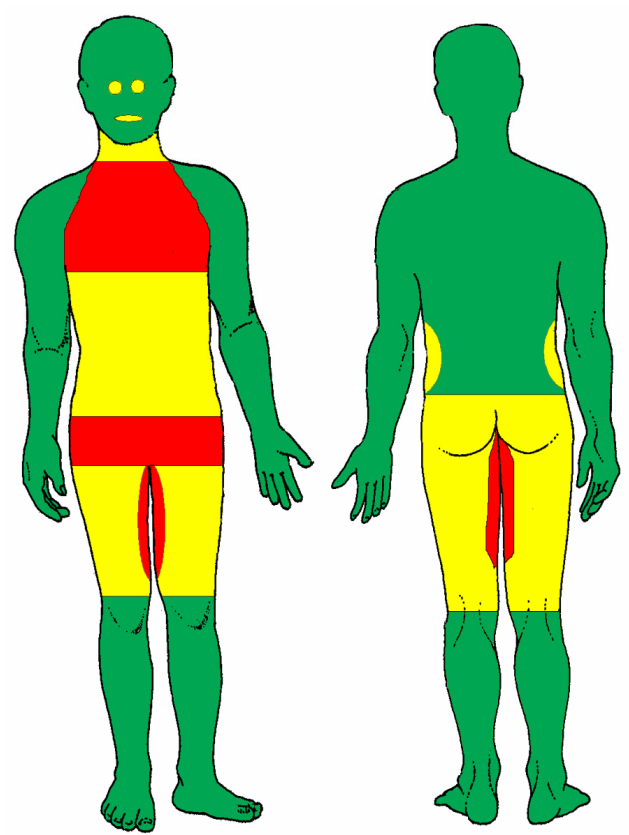

A-1d. Female beliefs about where other females may touch their bodies.

Appendix A-1

Residential University. Same-Gender Touch Zones [Public=green (medium shade); Discretionary=yellow (light shade); Private=red (dark shade)] $(\mathrm{N}=242)$ 


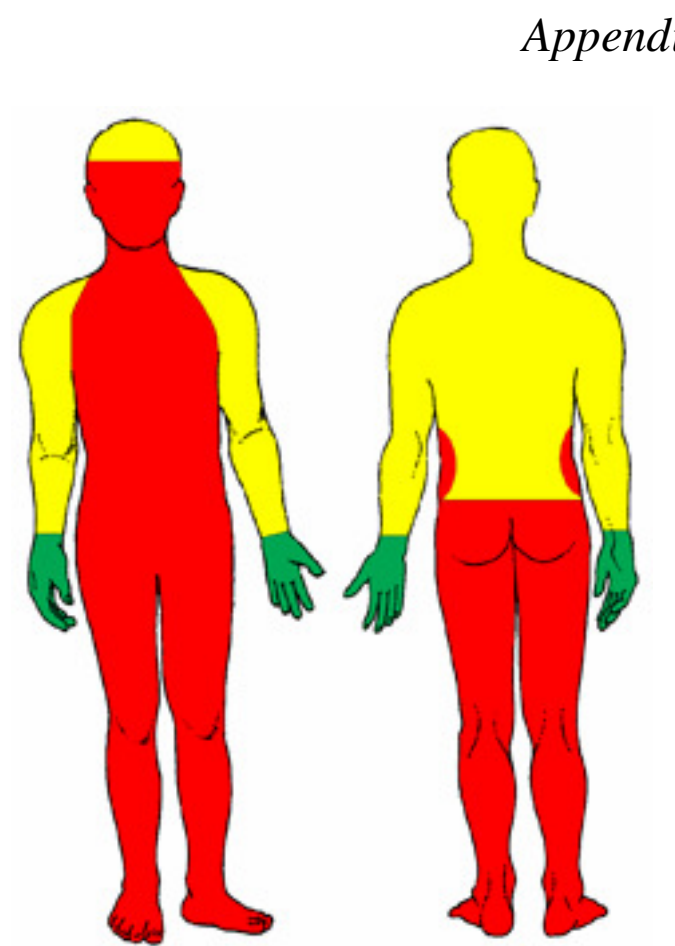

A-2a. Male beliefs about where they may touch other male bodies.

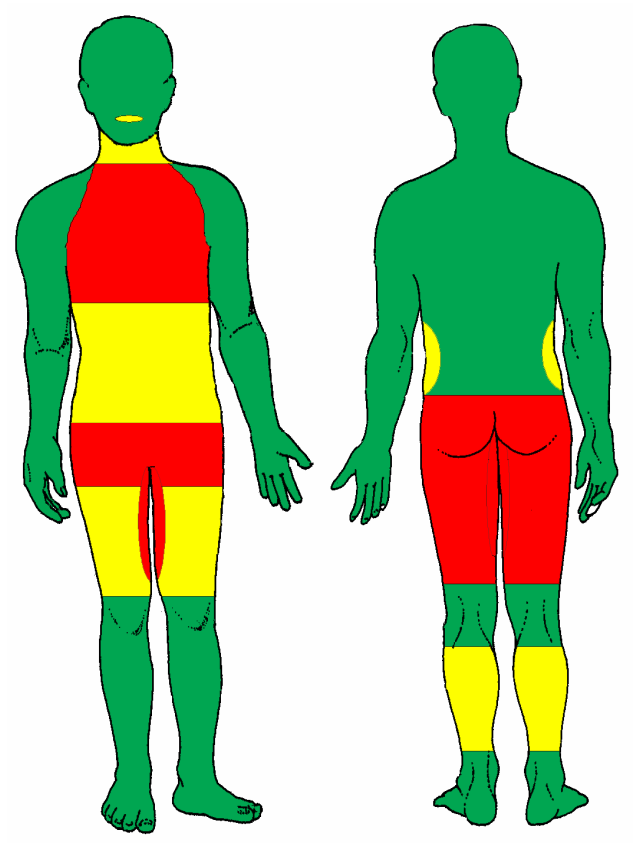

A-2c. Female beliefs about where they may touch other female bodies.

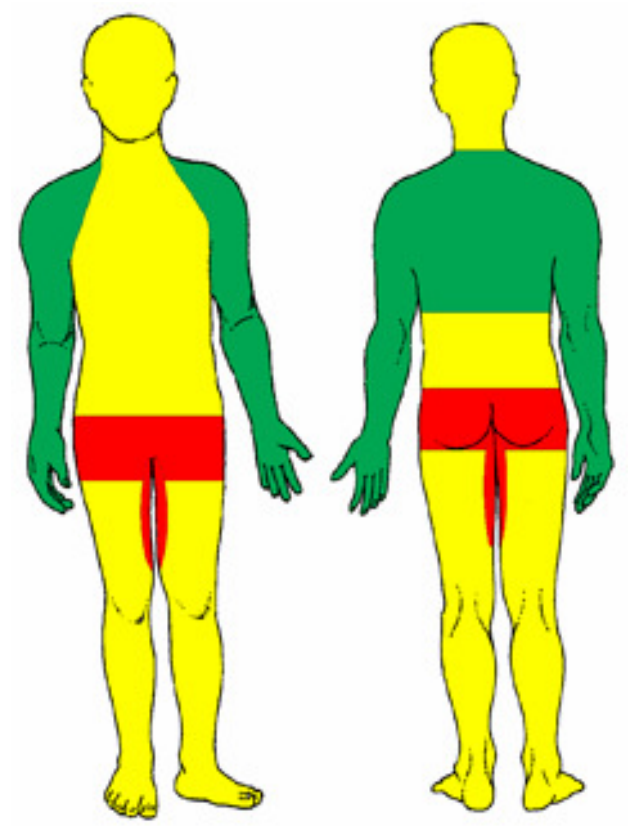

A-2b. Male beliefs about where other males may touch their bodies.

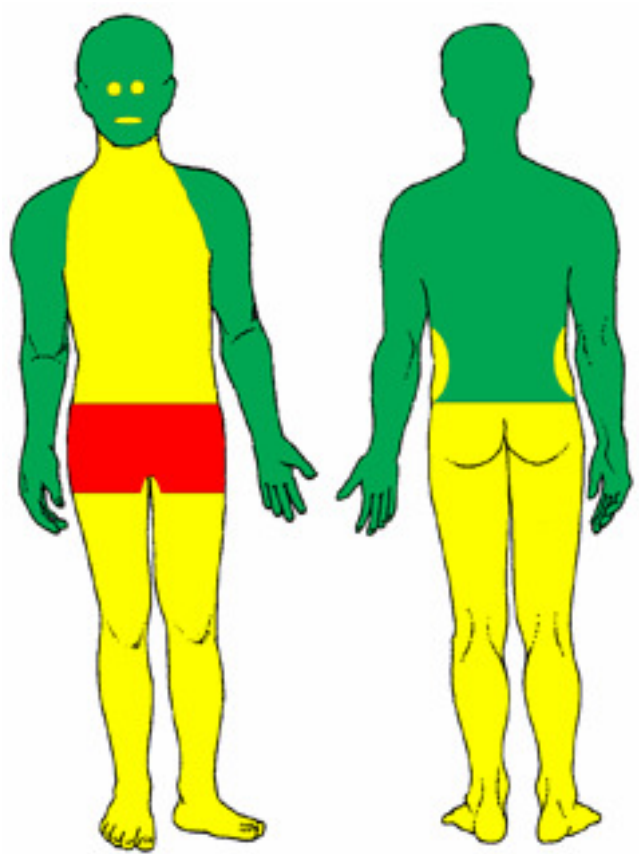

A-2d. Female beliefs about where other females may touch their bodies.

Appendix A-2

Community College. Same-Gender Touch Zones [Public=green (medium shade); Discretionary=yellow (light shade); Private=red (dark shade) $(\mathrm{N}=200)$ 


\section{Appendix B}

Opposite-Gender Touch Zones

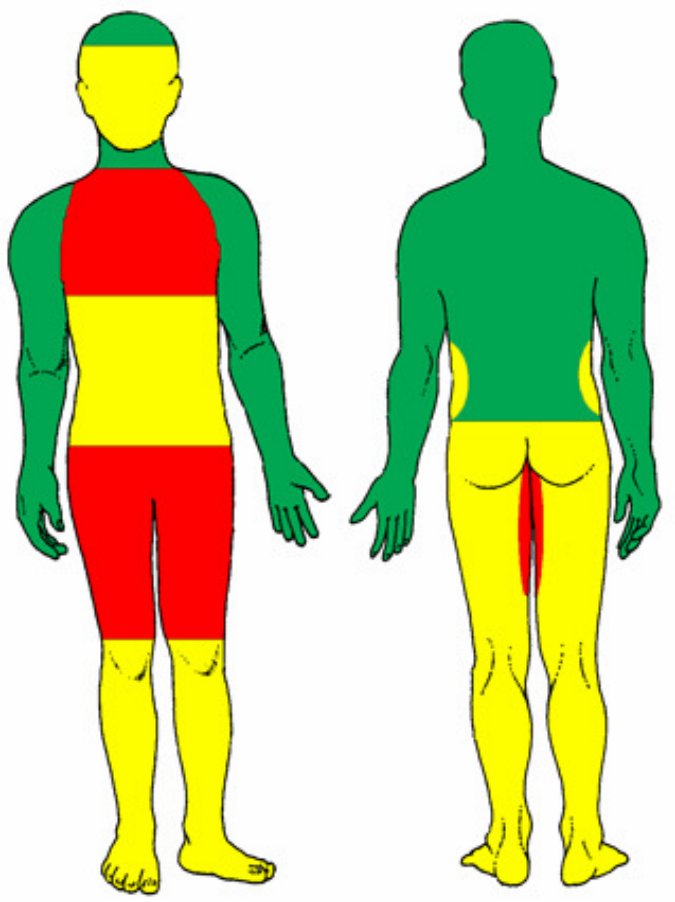

B-1a. Male beliefs about where they may touch female bodies.

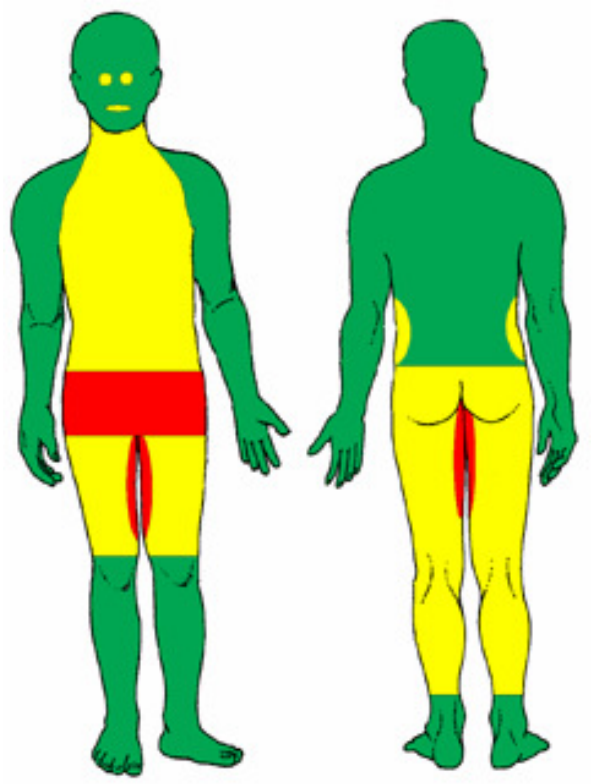

B-1c. Female beliefs about where they may touch male bodies.

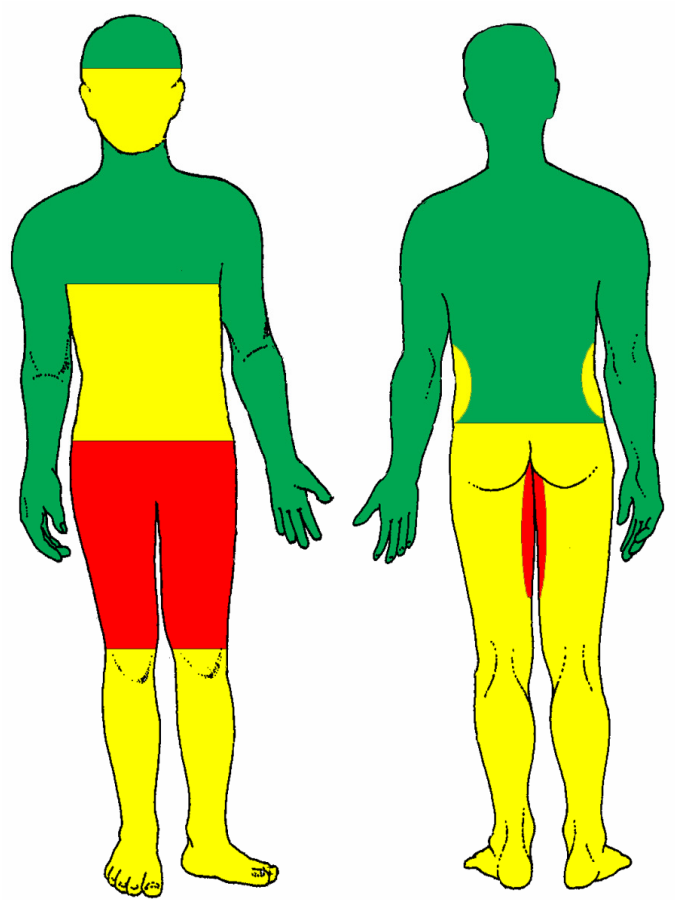

B-1b. Male beliefs about where females may touch their bodies.

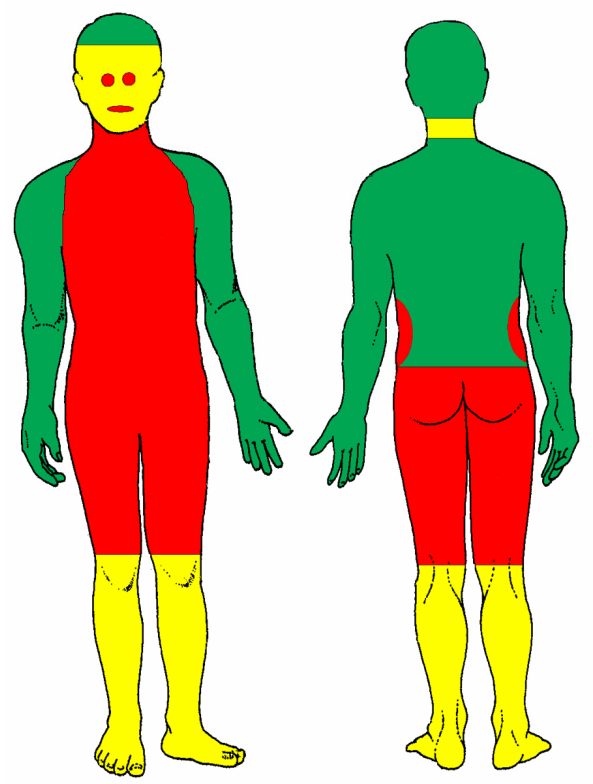

B-1d. Female beliefs about where males may touch their bodies.

Appendix B-1

Residential University Opposite-Gender Touch Zones [Public=green (medium shade); Discretionary=yellow (light shade); Private=red (dark shade)] $(\mathrm{N}=242)$ 


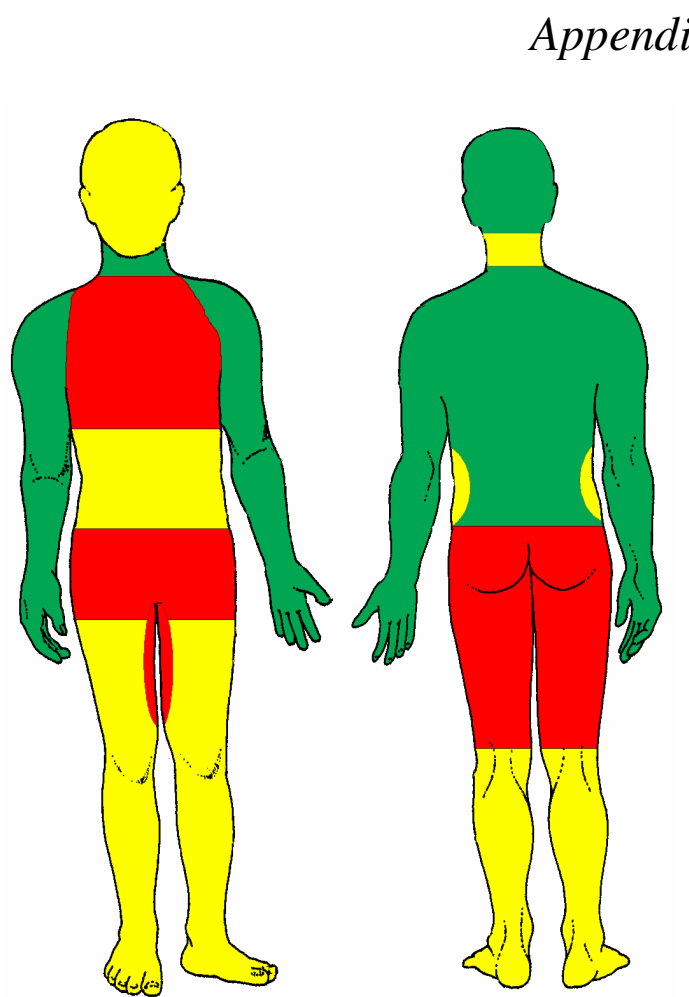

B-2a. Male beliefs about where they may touch female bodies.

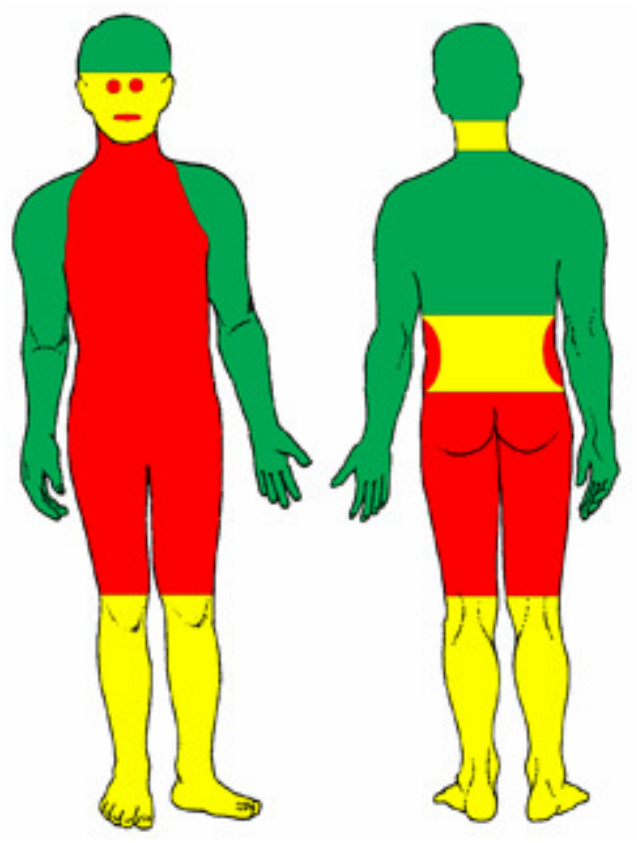

B-2c. Female beliefs about where they may touch male bodies.

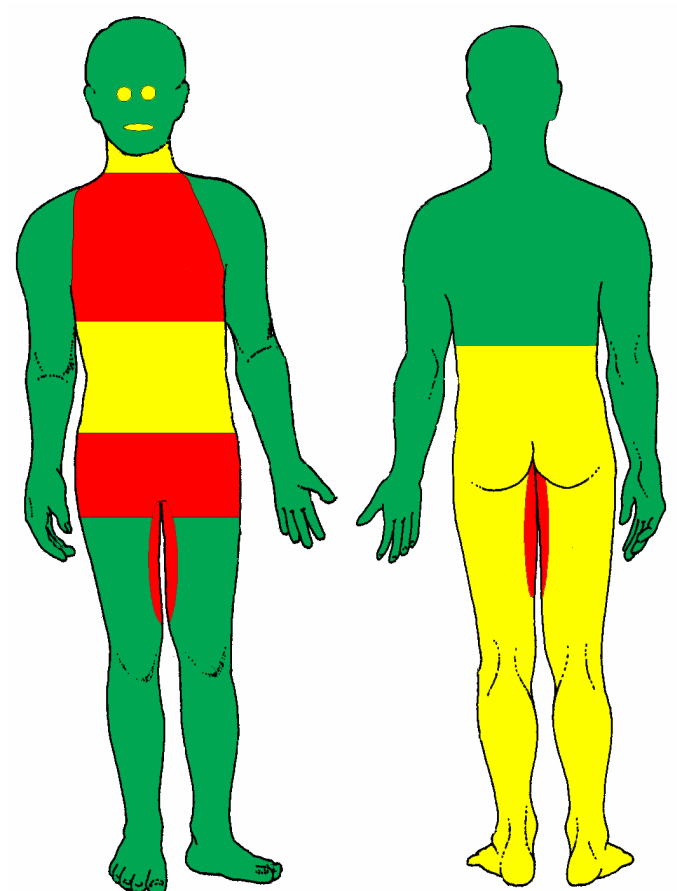

B-2b. Male beliefs about where females may touch their bodies.

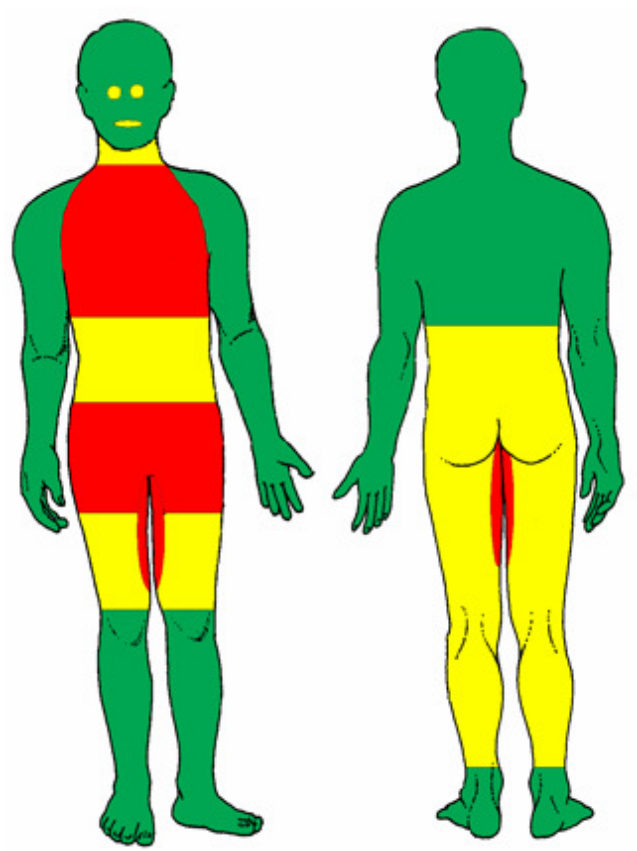

B-2d. Female beliefs about where males may touch their bodies.

Appendix B-2

Community College. Opposite-Gender Touch Zones [Public=green (medium shade); Discretionary=yellow (light shade); Private $=$ red (dark shade)] $(\mathrm{N}=200)$ 


\section{Appendix C}

Touch Zone Ratios: Residential University and Community College

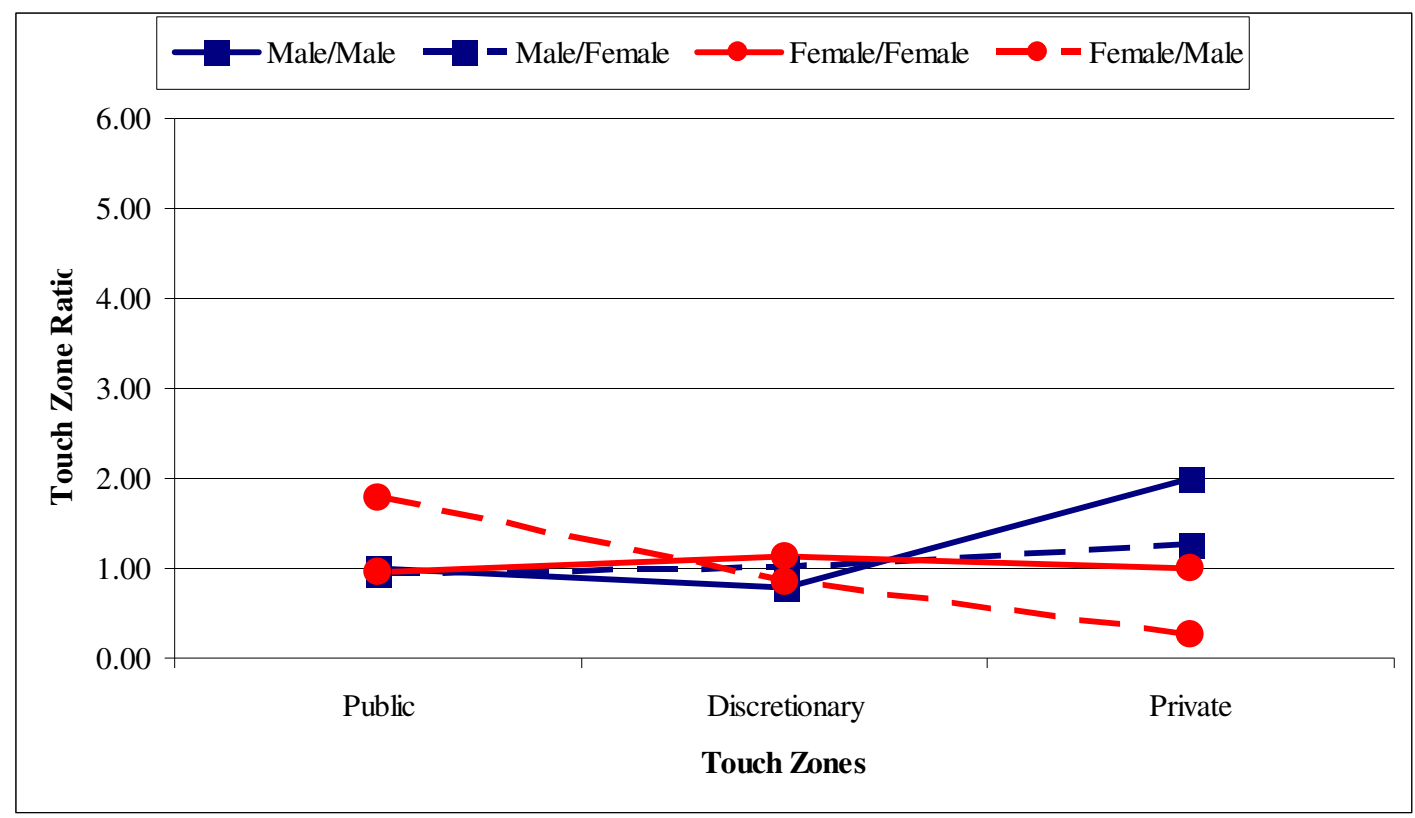

Figure 1A

Residential University. Touch zone ratios for public, discretionary, and private touch zones by gender and direction of touch $(\mathrm{N}=242)$.

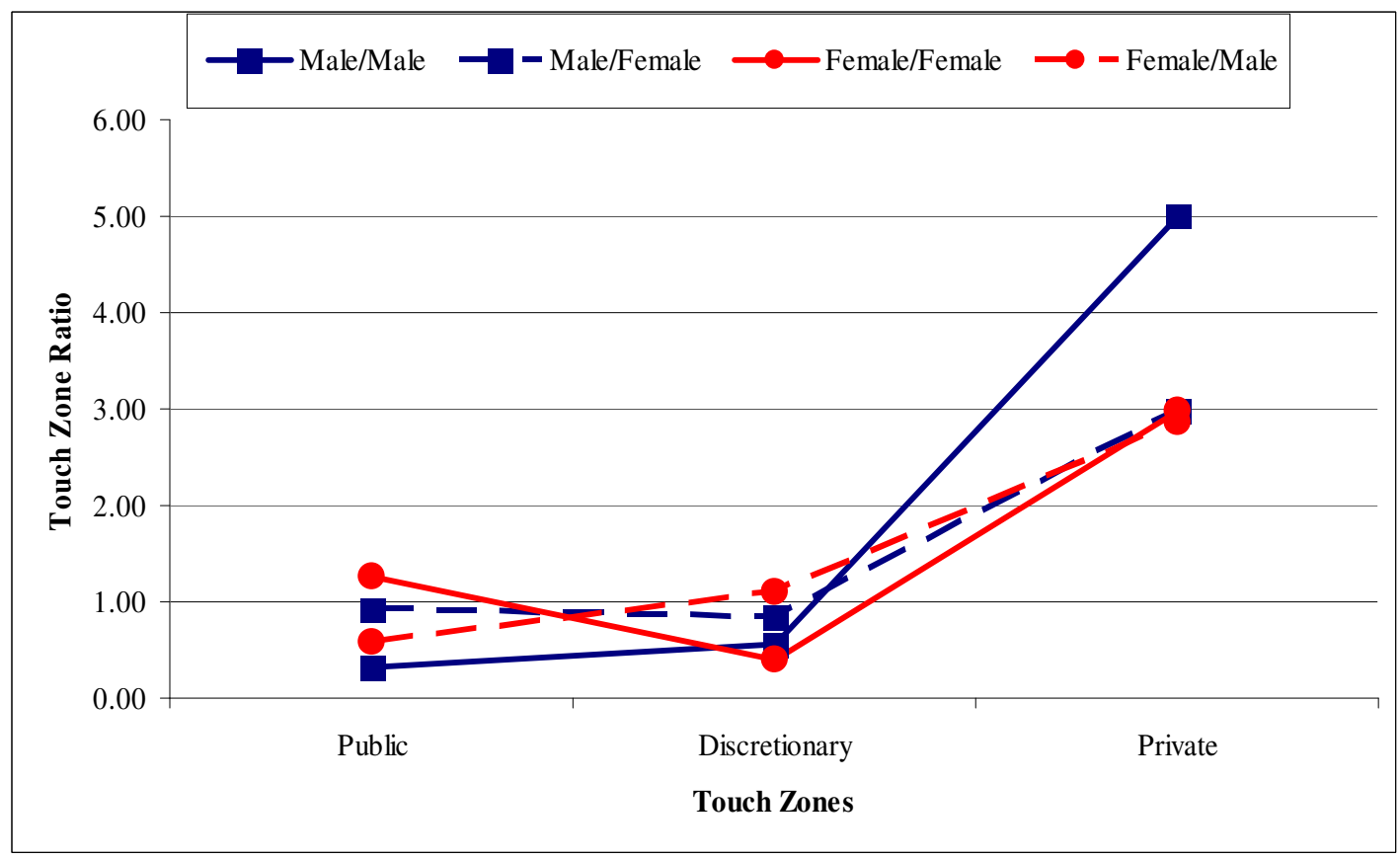

Figure 1B

Community College. Touch zone ratios for public, discretionary, and private touch zones by gender and direction of touch $(\mathrm{N}=200)$. 